Relations industrielles

Industrial Relations

\title{
Leclerc, Michel et Michel Quimper, Les relations du travail au Québec: une analyse de la situation dans le secteur public
}

\section{Jean Boivin}

Volume 49, numéro 2, 1994

URI : https://id.erudit.org/iderudit/050946ar

DOI : https://doi.org/10.7202/050946ar

Aller au sommaire du numéro

Éditeur(s)

Département des relations industrielles de l'Université Laval

ISSN

0034-379X (imprimé)

1703-8138 (numérique)

Découvrir la revue

Citer ce compte rendu

Boivin, J. (1994). Compte rendu de [Leclerc, Michel et Michel Quimper, Les relations du travail au Québec : une analyse de la situation dans le secteur public]. Relations industrielles / Industrial Relations, 49(2), 408-411.

https://doi.org/10.7202/050946ar

Tous droits réservés (C) Département des relations industrielles de l'Université Laval, 1994
Ce document est protégé par la loi sur le droit d'auteur. L'utilisation des services d'Érudit (y compris la reproduction) est assujettie à sa politique d'utilisation que vous pouvez consulter en ligne.

https://apropos.erudit.org/fr/usagers/politique-dutilisation/ 
La troisième partie du livre porte sur le « comment faire ". Elle aborde les questions de la préparation à la mise en place d'un comité, de son fonctionnement (le planifier, entraîner les parties à le gérer, etc.) et de son évaluation. Les auteurs se défendent d'avoir écrit un livre de recettes puisque chaque application peut varier par rapport à une autre; il s'agit plutôt de soulever les questions à se poser face à chaque nouvel essai. Dans cette partie du livre, les conseils donnés proviennent encore d'une solide expérience des auteurs comme consultants.

La dernière partie du livre s'intéresse aux systèmes alternatifs de rémunération visant à « partager le gâteau ». L'implication des salariés dans les nouvelles expériences de travail contribue souvent à reconsidérer les modèles traditionnels de rémunération. Sont ainsi présentées différentes formes plus ou moins nouvelles de rémunération tels le salaire aux pièces, individuel ou collectif, ou encore la rémunération basée sur les connaissances. On y présente ensuite certains plans américains de partage des profits et finalement certaines expériences de propriété d'entreprises par les travailleurs. Pour chaque modèle, une définition est apportée alors que les auteurs tentent d'en faire ressortir les aspects positifs et négatifs. Selon eux, une bonne paie ne suffit pas à créer un bon milieu de travail, pas plus que la participation seule n'est suffisante. Liés ensemble, ces deux éléments pourraient susciter, toujours selon les auteurs, une bonne « qualité de vie au travail ».

Cette référence assez constante dans le livre à la qualité de vie au travail, bien que les auteurs en parlent comme d'un concept générique, pourrait agacer certains syndicats, quand on sait le peu de succès que les programmes du même nom ont eu. Par ailleurs, comme on l'a dit souvent, les auteurs ont su faire une bonne place à la vision syndicale, surtout quand ils abordent les différents pièges des expériences présentées. Aussi, peut-on conclure qu'il s'agit d'un bon guide qui pourrait être utile autant à la partie syndicale qu'à la partie patronale dans les entreprises qui veulent mettre sur pied des programmes conjoints. Le livre présente de fait un très bon survol des différents concepts, il sait les illustrer à l'aide d'exemples et en faire ressortir les « pour » comme les « contre ». En ce sens, il peut aussi être utile à des étudiants ou étudiantes qui voudraient se familiariser avec ces notions et ces pratiques. Par contre, celui ou celle qui y chercherait une analyse poussée du phénomène de la participation patronale-syndicale ou des nouvelles formes d'organisation du travail serait déçu. D'ailleurs, la plus grande critique que l'on pourrait adresser à l'ouvrage serait sans doute ses lacunes au niveau de l'analyse. Mais s'il ne s'agit pas d'un livre de réflexion sur ces expériences, ça n'était pas là non plus l'intention première des auteurs.

Colette BERnier

Université Laval

Les relations du travail au Québec : une analyse de la situation dans le secteur public, par Michel Leclerc et Michel QuIMPER, Sainte-Foy, Presses de 1'Université du Québec, 1994, 336 p., ISBN 2-7605-0723-8.

Les auteurs présentent cet ouvrage comme « un survol rapide des relations du travail, en guise d'initiation générale et d'invitation à aller plus loin " (p. XIX). 
Cette remarque est importante car elle permet de comprendre le contenu plutôt descriptif qu'analytique du volume qui est divisé en quatre grandes parties : l'historique des relations du travail, la législation du travail, la vie syndicale au Québec et enfin, l'avenir des relations du travail. Le moins que l'on puisse dire est que les auteurs se sont donné un vaste champ à couvrir et on doit leur attribuer le mérite d'avoir su identifier les principales composantes du système québécois de relations industrielles. Par contre, on serait tenté de leur servir le proverbe " qui trop embrasse, mal étreint ", car il est pratiquement impossible de traiter sérieusement un sujet aussi vaste dans un seul volume de 336 pages.

En effet, l'histoire des relations du travail, objet des quatre premiers chapitres, porte sur rien de moins que l'Angleterre, la France, les États-Unis, le Canada et le Québec et ce, de la Révolution industrielle à nos jours! La législation du travail, pour sa part, est résumée dans quatre chapitres : d'abord le cadre constitutionnel, soit le partage des compétences en droit du travail et les chartes canadienne et québécoise des droits et libertés (chapitre 5); ensuite, les lois du travail autres que le Code, soit la Loi sur les normes du travail, la Loi sur les accidents du travail et les maladies professionnelles et la Loi sur la santé et la sécurité du travail (chapitre 6); puis, le Code du travail (chapitre 7); enfin, les secteurs public et parapublic (chapitre 8).

Trois chapitres composent la troisième partie : les organisations syndicales et patronales (chapitre 9), la négociation collective (chapitre 10) et la gestion de la convention collective (chapitre 11). Enfin, on retrouve également trois chapitres dans la dernière partie portant sur l'avenir des relations du travail : le chapitre 12 s'intitule « Les nouveaux programmes de gestion des ressources humaines $"$, le chapitre 13, "L'adaptation aux nouvelles réalités » et le chapitre 14, « Prospectives des relations du travail ».

Si les auteurs n'avaient pas pris la précaution de dire que ce livre contenait la matière première d'un cours donné par l'un des deux auteurs, il aurait été difficile de déterminer à quel public il était destiné étant donné la très grande diversité des sujets traités. Pour cette raison, il est sans doute à peu près impossible de l'utiliser comme lecture obligatoire dans le cadre d'un enseignement autre qu'un cours d'introduction générale aux relations du travail s'apparentant à celui auquel il est fait référence plus haut. Par exemple, raconter en 72 pages l'histoire des relations du travail dans quatre pays à partir de la Révolution industrielle jusqu'à nos jours implique nécessairement que les auteurs aient dû prendre des raccourcis qui les ont forcés à mettre de côté des événements importants. Il n'y a alors aucune utilité à faire lire ce texte à des étudiants et des étudiantes inscrits à un cours d'Histoire du syndicalisme.

Il en est de même pour la deuxième partie qui porte sur les principales lois ayant un impact sur les relations du travail. On consacre environ 60 pages à décrire le contenu de six textes législatifs mais le lecteur cherche en vain une quelconque analyse de la portée des dispositions les plus importantes de ces lois. Peut-on alors concevoir l'utilité d'un tel volume dans un cours de Droit du travail?

Il est encore plus surprenant de constater que même le chapitre consacré aux secteurs public et parapublic subit un traitement identique : on y décrit à fond le contenu des articles du Code du travail se rapportant à ces secteurs de même que ceux de la Loi 
sur les régimes de négociation des conventions collectives dans les secteurs public et parapublic mais on ne retrouve aucun jugement ni évaluation des faits vécus au cours des trente dernières années. Pourtant, le sous-titre du volume ne laisse aucun équivoque :Une analyse de la situation dans le secteur public. De plus, j'ai éprouvé beaucoup de difficultés à suivre la logique de présentation des données et ceci parce que les auteurs n'ont pas bien précisé au début du chapitre la distinction qui est faite dans le Code entre « services publics " et « secteur public ». Un lecteur non familier avec le domaine aura bien de la difficulté à comprendre la discussion concernant les services essentiels et le rôle différent joué par le Conseil du même nom selon qu'il s'agisse du secteur des affaires sociales ou des autres services publics.

Dans la troisième partie qui s'intitule « La vie syndicale au Québec », le premier chapitre donne des statistiques intéressantes sur les effectifs syndicaux et présente les organigrammes des centrales. Encore là, on aurait apprécié une certaine référence à l'évolution idéologique de ces organisations ou encore à certains traits caractéristiques des différentes centrales comme le fait que la FTQ possède une structure qui donne un pouvoir de réprésentation plus grand aux secteurs professionnels qu'aux conseils du travail alors qu'à la CSN, c'est le contraire; ou encore une allusion au fait que la CSD permet l'affiliation directe de la plupart des syndicats locaux sans passer par les structures de services habituels que sont les fédérations professionnelles. Malheureusement, on ne retrouve rien de cela.

Le chapitre suivant porte sur la négociation collective. Il décrit d'abord les principaux éléments du processus de négociation, de la préparation des parties à la dynamique même de l'action, tout en mentionnant les quatre conditions de Marshall qui influencent le pouvoir de négociation. Il présente aussi le modèle de Kochan et Barocci illustrant les influences réciproques du contexte externe et des stratégies utilisées par les parties. Il s'agit là, en somme, des éléments théoriques fondamentaux qui sont impliqués dans toute négociation collective.

Les auteurs débordent ensuite sur le secteur public et c'est là que j'éprouve certains problèmes. Leclerc et Quimper s'appuient sur un modèle de négociation multilatérale développé par Thomas Kochan à partir de l'expérience municipale américaine. Malgré tout le respect que j'éprouve pour ce célèbre chercheur américain et la valeur de son modèle, je crois que les auteurs ont raté une excellente occasion de nous parler de ce qu'ils nous avaient annoncé dans le titre même de leur volume, Les relations $d u$ travail auQuébec : une analyse de la situation dans le secteur public. Qui plus est, dans la dernière section qui s'intitule «L'avenir de la négociation collective ", les auteurs concluent sur une citation d'un auteur américain qui souligne la contradiction actuelle dans laquelle se retrouvent les relations du travail : " alors que dans certains cas on retrouve de véritables efforts de coopération patronale-syndicale à tous les niveaux de l'entreprise tandis que dans d'autres, certains efforts de coopération sur les lieux de travail sont déployés dans des entreprises où les dirigeants poursuivent une politique de confrontation avec les syndicats au niveau corporatif » (p. 231). Pourquoi citer un auteur américain dont le jugement est basé sur l'expérience américaine où le syndicalisme est en chute libre? Pourquoi ne pas plutôt discuter de la problématique « coopération/ confrontation » dans le contexte québécois alors que le syndicalisme est encore en santé et que les expériences de négociation de "contrats sociaux " se multiplient depuis 
quelques années. D'ailleurs, les expériences de fausse coopération auxquelles la citation fait référence sont loin d'être aussi répandues au Québec qu'aux États-Unis et, même si on pouvait en trouver, elles ne pourraient certainement pas être utilisées pour démontrer les pratiques ayant cours actuellement dans le secteur public alors que la problématique de la coopération en est encore (au mieux) à ses premiers balbutiements.

Le dernier chapitre de cette partie concerne la gestion de la convention collective et c'est nettement le meilleur du volume. On y retrouve un contenu détaillé qui réflète bien l'expérience pratique pertinente des deux auteurs.

Le contenu de la quatrième partie, "L'avenir des relations du travail ", est excellent mais l'ordre de présentation des trois chapitres crée beaucoup de confusion. Les auteurs ont choisi de débuter avec un chapitre sur « Les nouveaux programmes de gestion des ressources humaines ", suivi d'un autre intitulé " L'adaptation aux nouvelles réalités " et, enfin, "Perspectives des relations du travail ».

En débutant cette partie par une présentation de la panoplie des programmes de gestion des ressources humaines (santé et sécurité au travail, accès à l'égalité, politiques concernant les personnes atteintes du sida, gestion de carrière, aide aux employés, gestion de la performance, participation des travailleurs, qualité de vie au travail, cercles de qualité, partenariat, communication et qualité totale), les auteurs opèrent un changement de paradigme qui prend le lecteur par surprise, d'autant plus qu'ils n'ont pas situé au début du volume la place de la GRH par rapport aux relations du travail.

Une telle omission aurait été moins lourde de conséquence pour la compréhension de la démarche des auteurs si le chapitre intitulé «Perspectives de relations du travail » avait précédé celui sur les programmes de GRH. En effet, comme ce chapitre traite de l'impact des bouleversements profonds survenus dans le contexte économique mondial sur la main-d'œuvre, l'emploi, le contexte organisationnel, la gestion et le syndicalisme, il aurait en quelque sorte servi d'entrée en matière à la description des programmes de gestion des ressources humaines qui revêtent de plus en plus d'importance au sein des entreprises.

Ce livre peut être fort utile à des personnes non familières avec le système de relations industrielles québécois et qui veulent obtenir une information générale sur ses principales composantes. Il ne peut malheureusement pas servir d'ouvrage de référence pouvant permettre à quelqu'un de se faire une opinion critique sur son fonctionnement.

Jean Borvin

Université Laval

Le Pouvoir et la Règle - Dynamiques de l'action organisée, par Erhard FRIEDBERG, Paris, Éditions du Seuil, 1993, 405 p., ISBN 2-02-019655-7.

Michel Crozier et Erhard Friedberg nous livraient en 1977, avec L'acteur et le système, une référence désormais incontournable en sociologie des organisations. Ils nous proposaient, avec l'analyse stratégique, un cadre original pour interpréter les relations sociales au travail. En étroite parenté, Le Pouvoir et la Règle pousse désormais la 